\title{
Acetylene Reactivity on Pd-Cu Nanoparticles Supported on Thin Silica Films: The Role of the Underlying Substrate
}

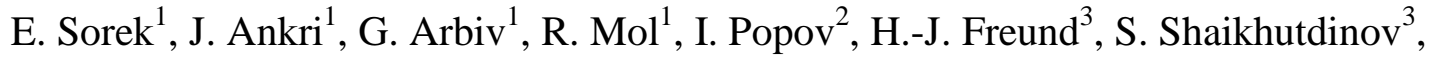 \\ and M. Asscher ${ }^{1 *}$ \\ 1. Institute of Chemistry, Edmond J. Safra Campus, The Hebrew University of Jerusalem, Givat- \\ Ram Jerusalem, Israel \\ 2. The Harvey M. Krueger Family Center for Nanoscience and Nanotechnology, The Hebrew \\ University, Jerusalem, 91904, Israel \\ 3. Fritz-Haber Institute der Max-Planck Gesellschaft, Department of Chemical Physics, \\ Faradayweg 4-6, D-14195 Berlin, Germany
}

\section{Supporting Information}

The $\mathrm{Pd}-\mathrm{Cu}$ nanoparticles were grown on top of the bilayer $\mathrm{SiO}_{2} / \mathrm{Ru}(0001)$ substrate by the Buffer Layer Assisted Growth (BLAG) mechanism in which $\mathrm{Pd}$ and $\mathrm{Cu}$ atoms were co-deposited on a $20 \mathrm{ML}$ layer of amorphous solid water (ASW) at 110K. Post deposition TEM analysis of the nanoparticles revealed that the elemental ratio determined in-situ in the vacuum chamber utilizing a quartz micro balance instrument, which was at a 1:1 ratio, was indeed verified by ex-situ TEM/EDS analysis of the elements on a single particle. In addition, the crystalline structure of the bimetallic clusters was shown to represent the d-spacing of the bi-metallic alloy (2.167 or $2.129 \AA$ in the two spots that were examined in the image below in Figure S1) compared to the literature values of $2.246 \AA$ for clean $\mathrm{Pd}$ and $2.088 \AA$ for clean $\mathrm{Cu}$. The measured value of the alloy NPs is thus almost exactly the average value of that of pure $\mathrm{Pd}$ and of pure $\mathrm{Cu}$ crystals, as shown below in Table S1. Important to note that unlike benzene, butene $\left({ }^{13} \mathrm{C}_{4} \mathrm{H}_{4}\right)$ at mass 56 was not found among the products. Apparently, this is an unstable product that undergoes rapid decomposition if it forms at all. 


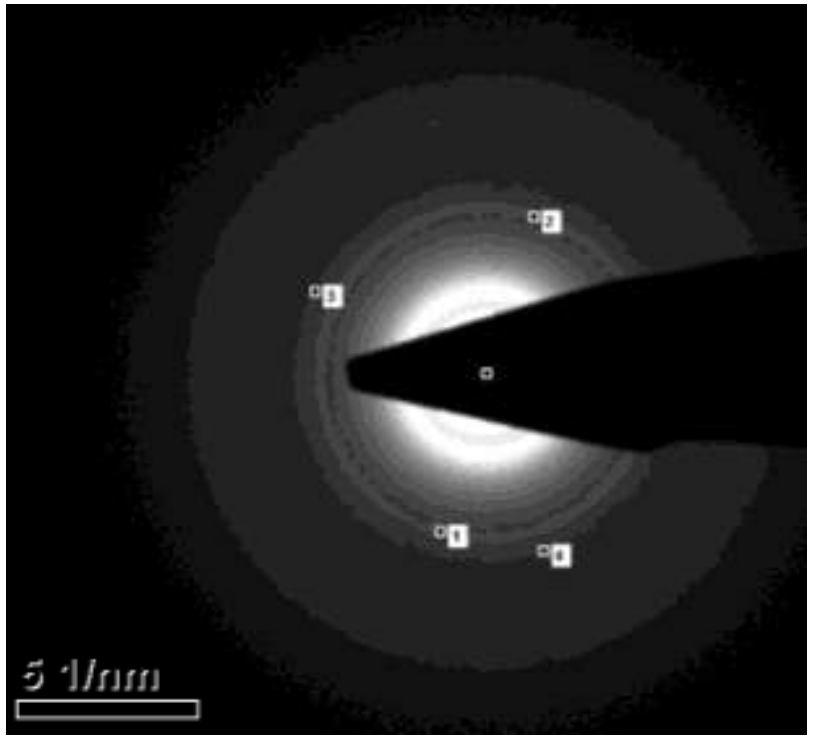

Figure S1: Diffraction pattern taken from the bimetallic Pd-Cu clusters, as shown in Figure 4A in the main text. Spots are listed in Table S1.

Table S1: Experimental and literature d-spacing values obtained from the diffraction image shown in Figure S1.

\begin{tabular}{|c|c|c|c|c|c|}
\hline \multirow[b]{2}{*}{ Spot\# } & \multirow{2}{*}{$\begin{array}{c}\text { Crystal } \\
\text { plane }\end{array}$} & \multirow{2}{*}{$\begin{array}{l}\text { Experimental } \\
\text { d-Spacing }(\AA)\end{array}$} & \multicolumn{3}{|c|}{ Literature d-Spacing $(\AA)$} \\
\hline & & & Pd & $\mathbf{C u}$ & $\begin{array}{l}\text { Pd-Cu } \\
\text { Alloy }\end{array}$ \\
\hline 1 & \multirow{2}{*}{$(111)$} & 2.129 & \multirow{2}{*}{2.246} & \multirow{2}{*}{2.088} & \multirow{2}{*}{2.178} \\
\hline 2 & & 2.167 & & & \\
\hline 3 & \multirow{2}{*}{ (200) } & 1.874 & \multirow{2}{*}{1.945} & \multirow{2}{*}{1.808} & \multirow{2}{*}{1.886} \\
\hline 4 & & 1.883 & & & \\
\hline
\end{tabular}

It is evident that the experimental (111) plane d-spacing in spot $2(2.167 \AA)$ and the (200) plane d-spacing in spot $4(1.883 \AA)$ are very close to the corresponding literature values of $2.178 \AA$ and $1.886 \AA$ for a $1(\mathrm{Pd}): 1(\mathrm{Cu})$ alloy and the values are in between the pure $\mathrm{Pd}$ and $\mathrm{Cu}$ d-spacing values. 

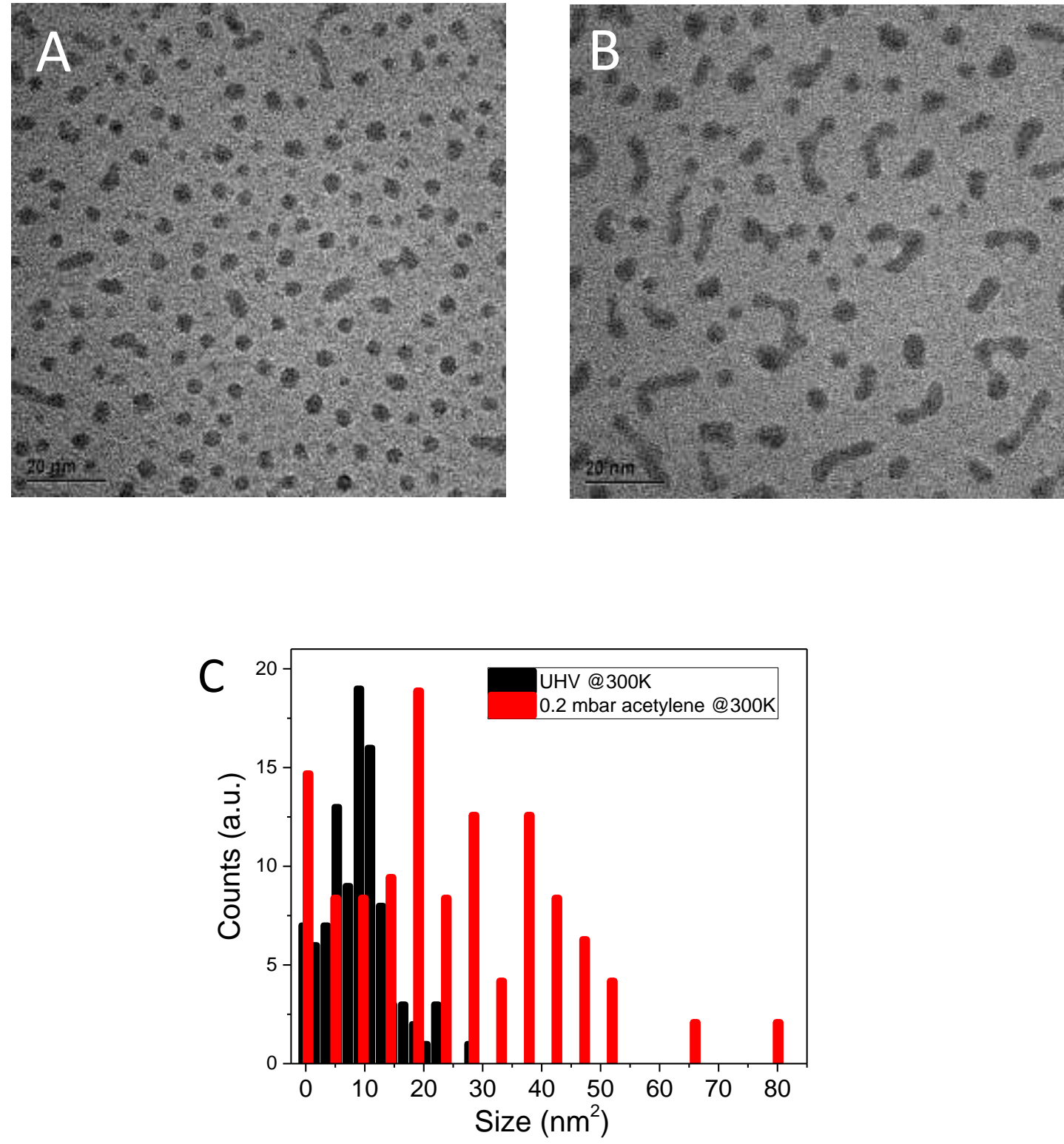

Figure S2: TEM images of Pd-Cu NPs grown on $\mathrm{SiO}_{2} / \mathrm{Cu}$ mesh (TEM substrate), deposited in UHV via BLAG: (A) NPs as prepared. (B) NPs exposed to 0.2 mbar at room temperature within the embedded medium-pressure cell. (C) $\mathrm{Pd}-\mathrm{Cu}$ bimetallic nanoparticles' size (area) distribution of the NPs shown in A (black bars) and B (red bars). 

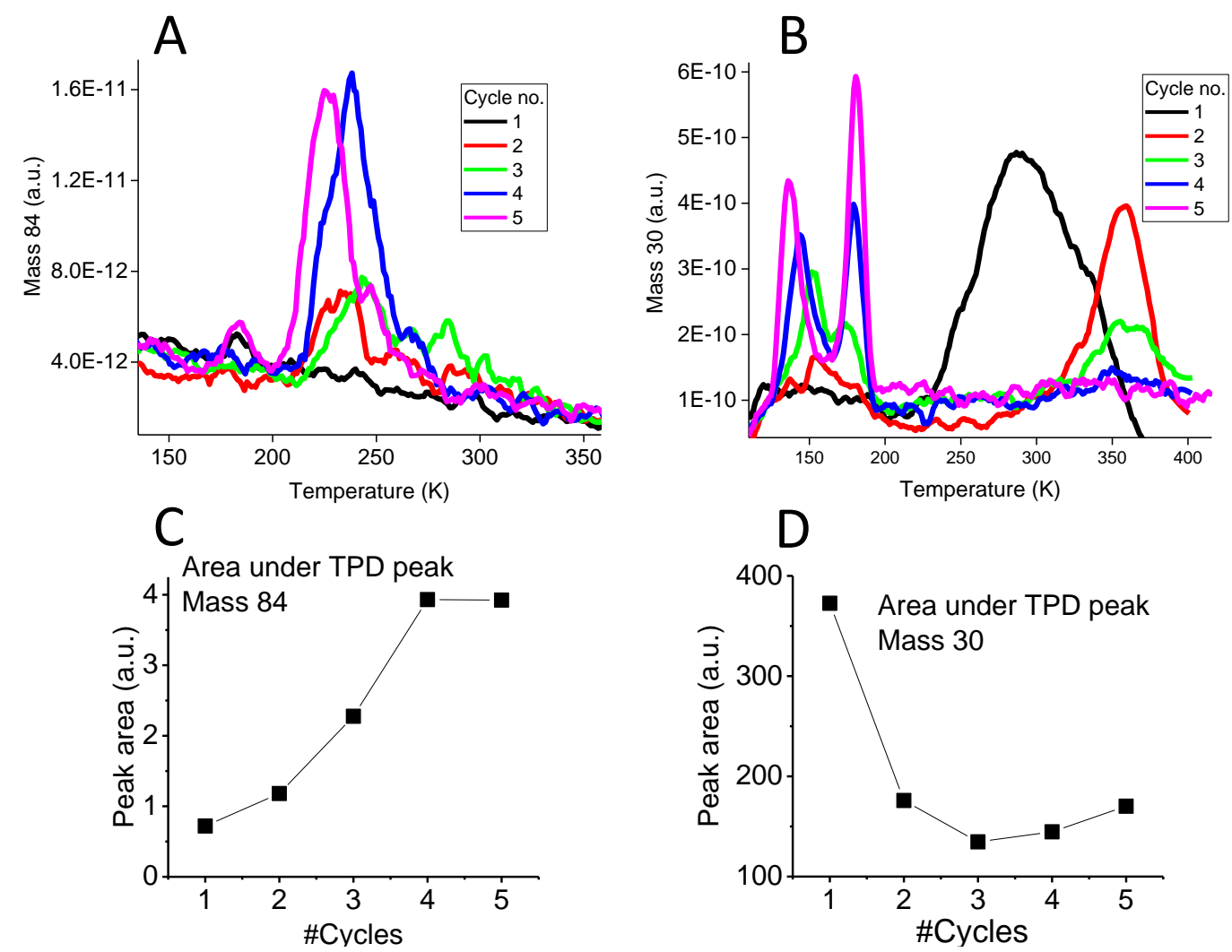

Figure S3: Temperature Programmed Reaction (TPR) spectra of the two products: (A) benzene at mass $84\left({ }^{13} \mathrm{C}_{6} \mathrm{H}_{6}\right)$ and (B) ethylene at mass $30\left({ }^{13} \mathrm{C}_{2} \mathrm{H}_{4}\right)$, obtained as a result of acetylene $\left({ }^{13} \mathrm{C}_{2} \mathrm{H}_{2}\right)$ catalytic decomposition following consecutive/repeated reaction cycles on the same substrate [(3Pd:3Cu) NPs/bilayer $\left.\mathrm{SiO}_{2} / \mathrm{Ru}(0001)\right]$. (C) and (D) are the corresponding integrated areas under the benzene and ethylene desorption peaks shown in A and B, respectively. Note the factor of 50 larger numbers in the case of ethylene compared to benzene. 

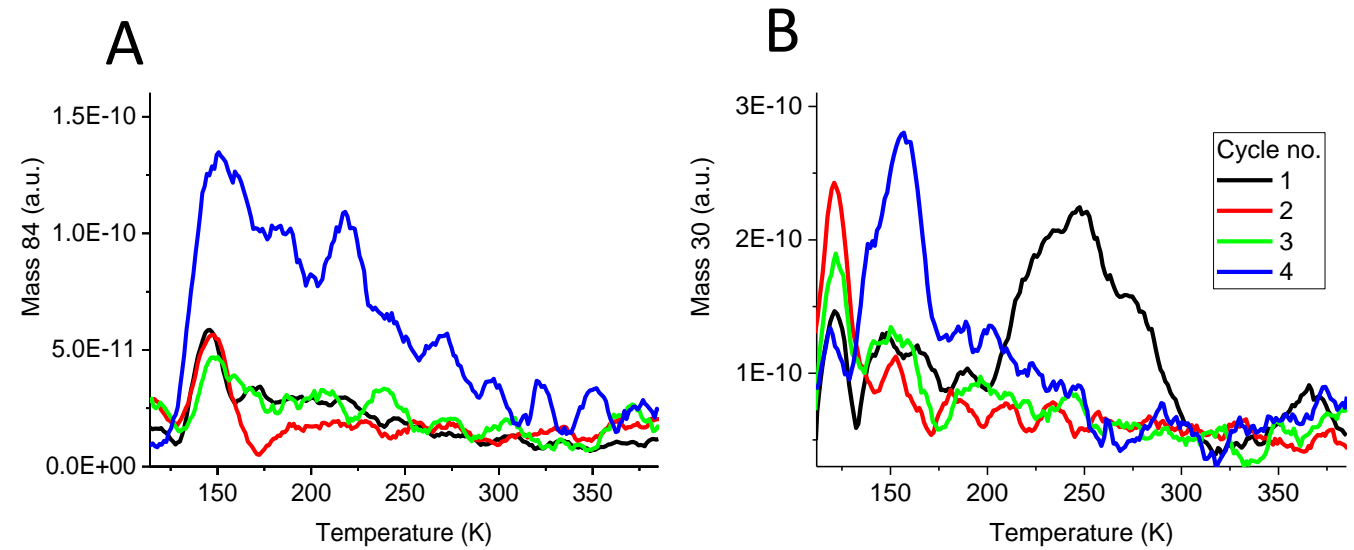

Figure S4: TPR measurements showing the production of ethylene and benzene following consecutive acetylene decomposition reactions on the same 1Pd:3Cu sample: (A) Mass 84 (benzene formation). (B) Mass 30 (ethylene formation)

Comparing the results of multiple TPR cycles of $3 \mathrm{Pd}: 3 \mathrm{Cu}$ (in Figure $\mathrm{S} 3$ ) to $1 \mathrm{Pd}: 3 \mathrm{Cu}$ shown in Figure S4 (as summarized in the main text, Figure 7), it is clear that the main effect here is the significant deactivation of the ethylene product as the number of cycles increases.

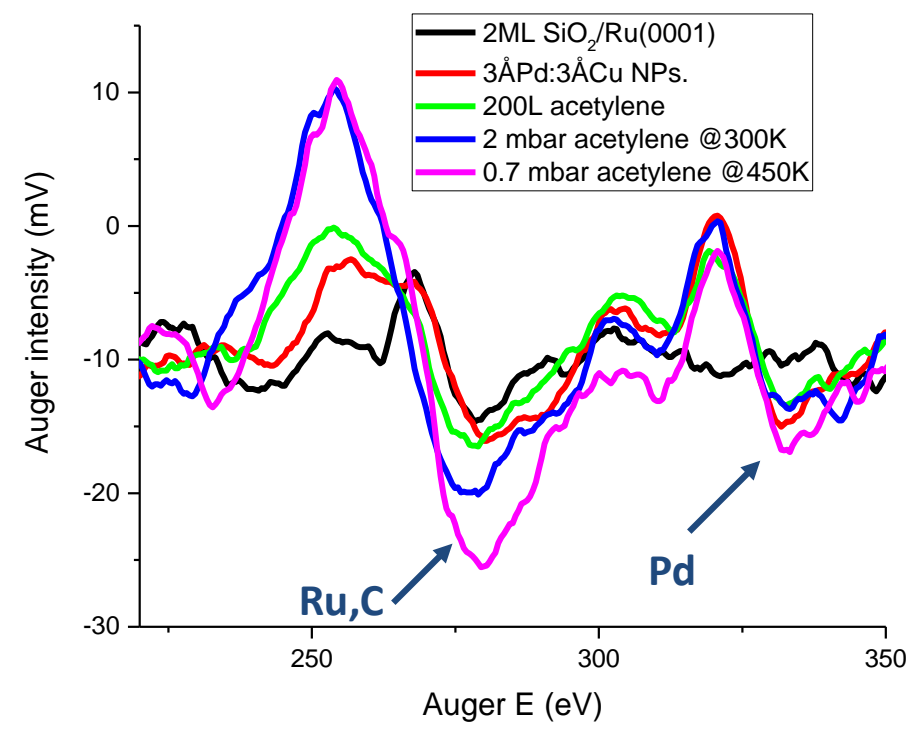

Figure S5: Auger spectra of the clean bilayer $\mathrm{SiO}_{2} / \mathrm{Ru}(0001)$ sample (black line) and the substrate with the bimetallic $\mathrm{Pd}-\mathrm{Cu}$ nanoclusters (red) and a growing exposure to acetylene at two temperatures, as indicated. 
The level of acetylene decomposition at 0.7 mbar @ 450K seems to be higher than at the higher pressure but at a lower temperature (2 mbar @ 300K). 